Methods: For this phenomenological study, narrative was obtained from eight Australian civilian hospital nurses following a disaster. Semi-structured interviews were conducted at two points in time. Descriptive moments of a possible lived experience were identified from participant narrative. These moments formed a lived experience description as an anecdote of an experience. A preparatory epoché-reduction and reduction proper was used to guide a reflection on the lived-space of being a nurse, following a disaster from the lived experience description. Results: Lived-space was described as shrinking then being open too-wide, where nurses were drawn into the disaster livedspace, then returning home to a wide-open but crowded lived-space. Disaster health lived-space was described as occupying, sharing and giving back.

Conclusion: This research provides insight into Australian civilian in-hospital nurse in the out-of-hospital disaster environment, following a disaster as part of a disaster medical assistance team. In particular this work adds a lived-space perspective to the existing literature. These insights may inform future education, research, clinical practice and policy.

Prehosp Disaster Med 2017;32(Suppl. 1):s146-s147

doi:10.1017/S1049023X17004046

\section{How Disaster Response in the Emergency Department Disrupts our Lived World \\ Karen Hammad ${ }^{1}$, Paul Arbon ${ }^{2}$, Kristine Gebbie ${ }^{2}$, Alison Hutton ${ }^{3}$}

1. Faculty Of Medicine, Nursing And Health Sciences, Flinders University, Adelaide/Australia

2. Torrens resilience Institution, Adelaide/SA/Australia

3. Faculty Of Medicine, Nursing And Health Sciences, Newcastle University, Newcastle upon Tyne/Australia

Study/Objective: According to van Manen (2015), there are five existential aspects common in the lived world of all humans. These include: "relationality" which is how we relate to others; "corporeality" how we experience our body; "temporality" our experience of time; "spatiality" how we experience space; and "materiality" our experience of things. This presentation will discuss how these existential aspects are experienced in the context of working as a nurse in the Emergency Department (ED) during a disaster.

Background: Our current understanding of emergency nurses and disaster response comes largely from narrative accounts which describe what it is like to work in the ED during a disaster response, or descriptive studies that explore emergency nurses' perceptions of as yet-hypothetical events. However, we have a limited understanding of how disaster response is experienced within the context of our existing lived world.

Methods: A Hermeneutic Phenomenological approach informed by van Manen underpins the research process. Thirteen nurses participated in this research. Thematic analysis and a guided existential reflection resulted in two different perspectives of the experience of working as a nurse in the ED during a disaster response. This presentation will report on the existential aspect of the research.

Results: Reflection on the five life existentials places a strong emphasis on the relational, spatial, and material aspects of disaster response, while corporeal and temporal aspects were minimised. Consideration of the existential aspects highlights how disaster response disrupts the everyday experience of working in the ED. The findings from this research can raise awareness for emergency nurses, and can inform preparedness for future disaster response.

Conclusion: This presentation focuses on one aspect of the findings of a $\mathrm{PhD}$ study and provides an in-depth insight into the meaning behind the experience of nursing in the ED during a disaster response.

Prehosp Disaster Med 2017;32(Suppl. 1):s147

doi:10.1017/S1049023X17004058

\section{Moments of Disaster Response in the Emergency Department Karen Hammad ${ }^{1}$, Paul Arbon ${ }^{2}$, Kristine Gebbie ${ }^{2}$, Alison Hutton $^{3}$}

1. Faculty Of Medicine, Nursing And Health Sciences, Flinders University, Adelaide/SA/Australia

2. Torrens resilience Institution, Adelaide/SA/Australia

3. Faculty Of Medicine, Nursing And Health Sciences, Newcastle University, Newcastle upon Tyne/SA/Australia

Study/Objective: "In the end, we will not remember the years we spent in nursing. We will only remember the moments" (Donahue 1996). We experience our lives as a series of memorable moments, some good and some bad. Undoubtedly, the experience of participating in disaster response is likely to stand out as a memorable moment in a nurses' career. This presentation will describe five distinct moments of nursing in the Emergency Department (ED) during a disaster response.

Background: Our existing understanding of emergency nurses' participation in disaster response comes largely from narrative accounts of single events. This study is unique because it explores the experience of nursing in the ED during a disaster across different geographical regions and disaster types.

Methods: A Hermeneutic Phenomenological approach informed by van Manen underpins the research process. Thirteen nurses from different countries around the world participated in interviews about their experience of working in the ED during a disaster. Interviews were conducted face to face or via Skype. Thematic analysis and a guided existential reflection resulted in two different perspectives of the experience of working as a nurse in the ED during a disaster response. This presentation will report on one of these aspects. Results: "The moments of notification, waiting, patient arrival, caring for patients, and reflection" described by nurses who participated in this research provide insight into the experience of nursing in the ED during a disaster response. Consideration of these individual moments will lead to recommendations for future preparedness of emergency nurses.

Conclusion: This presentation focuses on one aspect of the findings of a $\mathrm{PhD}$ study, and provides an in-depth insight into the experience of nursing in the ED during a disaster response, which can help generate awareness and inform future disaster preparedness of emergency nurses.

Prehosp Disaster Med 2017;32(Suppl. 1):s147

doi:10.1017/S1049023X1700406X 